\title{
Chiral symmetry breaking from five dimensional spaces
}

\author{
Leandro Da Rold* \\ Universitat Autònoma de Barcelona, Spain \\ E-mail: daroldleifae.es
}

\section{Alex Pomarol}

Universitat Autònoma de Barcelona, Spain

E-mail: alex.pomarolecern.ch

\begin{abstract}
Based on the AdS/CFT correspondence we study the breaking of the chiral symmetry in QCD using a simple five dimensional model. The model gives definite predictions for the spectrum of vector mesons, their decay constants and interactions as a function of one parameter related to the quark condensate. We calculate the coefficients $L_{i}$ of the low-energy QCD chiral lagrangian, as well as other physical quantities for the pions. All the predictions are shown to be in good agreement with the experimental data. We also show that they are robust under modifications of the 5D metric in the IR, and that some of them arise as a consequence of the higher-dimensional gauge symmetry. For example, at the tree-level, we find $M_{\rho} \simeq \sqrt{3} g_{\rho \pi \pi} F_{\pi}, F_{\rho} \simeq \sqrt{3} F_{\pi}$ and $\mathrm{BR}\left(a_{1} \rightarrow \pi \gamma\right)=0$.
\end{abstract}

International Europhysics Conference on High Energy Physics

July 21st - 27th 2005

Lisboa, Portugal

* Speaker. 


\section{Introduction}

The string/gauge duality [1] has allowed us in the last years to gain new insights into the problem of strongly coupled gauge theories. Although a string description of real QCD has not yet been formulated, different string constructions have been able to describe gauge theories with certain similarities to QCD, such as confinment and chiral symmetry breaking. The incorporation of fundamental quarks as D7-branes in the $\mathrm{AdS}_{5} \times \mathrm{S}^{5}$ background [2] has allowed to address flavor issues (see references in [3]). A more phenomenological approach to QCD consists in using 5D field theories in Anti-de-Sitter (AdS). This is a more modest attempt but, in certain regimes, it grasps the generic features of the more involved string constructions.

We propose a simple model to study chiral symmetry breaking [3] in the vector and axialvector sector of QCD (the same model was proposed in [4]). It is known from the OPE that the vector-vector current correlator for large Euclidean momentum, $p \gg \Lambda_{\mathrm{QCD}}$, is given in the chiral limit by [5]

$$
\Pi_{V}\left(p^{2}\right)=p^{2}\left[\beta \ln \frac{\mu^{2}}{p^{2}}+\frac{\gamma}{p^{4}}+\frac{\delta}{p^{6}}+\cdots\right],
$$

where $\beta \simeq N_{c} /\left(12 \pi^{2}\right), \gamma \simeq \alpha_{s}\left\langle G_{\mu \nu}^{2}\right\rangle / 12 \pi$ and $\delta \simeq-28 \pi \alpha_{s}\langle\bar{q} q\rangle^{2} / 9$ are almost momentum-independent coefficients. Similar expression holds for the axial-axial correlator $\Pi_{A}$. Therefore QCD behaves in Eq. (1.1) as a near-conformal theory in the ultraviolet (UV) in which the breaking of the conformal symmetry is given by the condensates. The correlator $\Pi_{V}$, on the other hand, must have, according to the large- $N_{c}$ expansion, single poles in the imaginary axis of $p$ corresponding to colorless vector resonances. These properties of QCD can be implemented in a 5D theory in AdS. The condensates $\langle\mathscr{O}\rangle$ are described, in the AdS side, by vacuum expectation values (VEV) of scalars $\Phi$ whose masses are related to the dimension $d$ of $\mathscr{O}$ by [6] $d=\sqrt{4+M_{\Phi}^{2} L^{2}}+2(L$ is the AdS curvature radius), while confinement and the mass gap in QCD can be obtained in the $\mathrm{AdS}_{5}$ by compactifying the fifth dimension. Alike large- $N_{c} \mathrm{QCD}$, the $5 \mathrm{D}$ theory is also described as a function of weakly coupled states corresponding to the mesons.

\section{A 5D model for chiral symmetry breaking}

The 5D analog of QCD with 3 flavors consists in a theory with a $\mathrm{SU}(3)_{L} \otimes \mathrm{SU}(3)_{R}$ gauge symmetry in the 5D bulk, a parity defined as the interchange $L \leftrightarrow R$ and a scalar field $\Phi$ transforming as a $\left(\mathbf{3}_{\mathbf{L}}, \overline{\mathbf{3}}_{\mathbf{R}}\right)$ whose VEV will be responsible for the breaking of the chiral symmetry. The 5D metric is defined generically as $d s^{2}=a^{2}(z)\left(\eta_{\mu \nu} d x^{\mu} d x^{\nu}-d z^{2}\right)$, where $a$ is the warp factor. We will work within $\mathrm{AdS}_{5}$, then the warp factor is given by $a(z)=L / z$, where $L$ is the AdS curvature radius. We will compactify this space by putting two boundaries, one at $z=L_{0}$ (UV-boundary) and another at $z=L_{1}$ (IR-boundary). The UV-boundary acts as a regulator necessary to obtain finite calculations. The limit $L_{0} \rightarrow 0$ should be taken after divergencies are canceled by adding counterterms on the UV boundary [6]. The IR-boundary is needed to introduce a mass gap in the theory $\sim 1 / L_{1}$ to be related to $\Lambda_{Q C D}$, and it breaks the conformal symmetry.

The action is given by

$$
S_{5}=M_{5} \int d^{4} x \int d z \sqrt{g} \operatorname{Tr}\left[-\frac{1}{4} L_{M N} L^{M N}-\frac{1}{4} R_{M N} R^{M N}+\frac{1}{2}\left|D_{M} \Phi\right|^{2}-\frac{1}{2} M_{\Phi}^{2}|\Phi|^{2}\right],
$$


where $D_{M} \Phi=\partial_{M} \Phi+i L_{M} \Phi-i \Phi R_{M}, L_{M}=L_{M}^{a} T^{a}$ and $\operatorname{Tr}\left[T^{a} T^{b}\right]=\delta_{a b}$, and similarly for the other fields. A coefficient $M_{5}$ has been factored out in front of the lagrangian so that $1 / \sqrt{M_{5}}$ is the $5 \mathrm{D}$ expansion parameter playing the role of $1 / \sqrt{N_{c}}$ in QCD. We define $\Phi=S e^{i P / v(z)}$ where $v(z) \equiv\langle S\rangle$.

We assume $M_{\Phi}^{2}=-3 / L^{2}$ that corresponds in the CFT to an operator of dimension 3 such as $\bar{q} q$. Solving the bulk equation of motion for $S$ we get $v(z)=c_{1} z+c_{2} z^{3}$, where $c_{1}$ and $c_{2}$ are two integration constants. They can be determined as a function of the value of $v(z)$ at the boundaries: $M_{q} \equiv L v /\left.z\right|_{L_{0}}$ and $\left.\xi \equiv L v\right|_{L_{1}}$. By the AdS/CFT correspondence, a nonzero $M_{q}$ is equivalent to put an explicit breaking of the chiral symmetry in the CFT (such as adding quark masses). A nonzero value of $\xi \propto \mathbb{I}$ corresponds in the chiral limit to an spontaneously breaking $\mathrm{SU}(3)_{L} \otimes \mathrm{SU}(3)_{R} \rightarrow \mathrm{SU}(3)_{V}$, playing the role of the condensate $\langle\bar{q} q\rangle$ in $\mathrm{QCD}$, as can be found by computing the variation of the vacuum energy with respect to $M_{q}$. We will take $\xi \rightarrow \xi \mathbb{I}$ where $\xi$ will be considered an input parameter. Therefore the vector sector of the model has 4 parameters, $M_{5}, M_{q}, L_{1}$, and $\xi$. The model then has, with respect to QCD, only one extra parameter, $\xi$.

Few comments: (1) Using naive dimensional analysis one can estimate that this 5D theory becomes strongly coupled at a scale $\sim 24 \pi^{3} M_{5}$. This implies that extra (stringy) physics must appear at this scale or, equivalently, that this is the scale that suppresses higher dimensional operators in Eq. (2.1). We estimate this scale to be around few GeV. (2) We are neglecting the backreaction on the metric due to the presence of the scalar VEV. Although a nonzero energy-momentum tensor of $\Phi$ will affect the geometry of the space producing a departure from AdS, this effect will only be relevant at $z$ very close to the IR-boundary, and therefore it will not substantially change our results. Notice that neglecting the backreaction corresponds to freeze other possible condensates that turn on in the presence of the quark condensate. (3) We will not consider the extra $\mathrm{U}(1)_{L, R}$ that involves the anomaly.

\subsection{The current-current correlators $\Pi_{V, A}$}

In QCD the generating functional of the current-current correlators is calculated by integrating out the quarks and gluons as a function of the external sources. This must be equivalent in the large$N_{c}$ limit to integrate all the colorless resonances at tree-level. The AdS/CFT correspondence tells us that this generating functional is the result of integrating out, at tree-level, the $5 \mathrm{D}$ gauge fields restricted to a given UV-boundary value $\left.V_{\mu}\right|_{L_{0}}=v_{\mu}$ and $\left.A_{\mu}\right|_{L_{0}}=a_{\mu}$ (with $V / A=(L \pm R) / \sqrt{2}$ ). Then $v_{\mu}\left(a_{\mu}\right)$ play the role of external source coupled to the vector (axial-vector) QCD current.

The two-point correlator $\Pi_{V}$ can be calculated analytically in terms of Bessel functions. For large Euclidean momentum, $p L_{1} \gg 1$, the dependence on $p$ of the correlators is dictated by the conformal symmetry and we find $\Pi_{V}\left(p^{2}\right) \simeq-M_{5} L p^{2} \ln \left(p L_{0}\right)$. Matching the coefficient $M_{5} L$ to the QCD $\beta$-function of Eq. (1.1). We get

$$
M_{5} L=\frac{N_{c}}{12 \pi^{2}} \equiv \tilde{N}_{c}
$$

that fixes the value of the $5 \mathrm{D}$ coupling. The correlator $\Pi_{A}$ depends on the $z$-dependent mass of $A_{\mu}$ and can be calculated analytically only in the large and small momentum limits. For $\xi \gg 1$ the dependence of $\Pi_{A}$ on $\xi$ is simply dictated by the conformal symmetry. For small $p$, we have

$$
\Pi_{A}\left(p^{2}\right)=F_{\pi}^{2}+\mathscr{O}\left(p^{2}\right), \quad \text { where } F_{\pi} \simeq 87\left(\frac{\xi}{4}\right)^{\frac{1}{3}} \mathrm{MeV}
$$


in excellent agreement with the experimental value for $\xi \simeq 4$. For large $p$ and in the chiral limit the left-right correlator $\Pi_{L R}=\Pi_{V}-\Pi_{A}$ is given by

$$
\Pi_{L R}\left(p^{2}\right) \simeq \frac{c_{6}}{p^{4}}+\cdots, \quad \text { where } \quad c_{6}=-\frac{16}{5} \frac{\tilde{N}_{c} \xi^{2}}{L_{1}^{6}} \simeq-1.4 \times 10^{-3}\left(\frac{\xi}{4}\right)^{2} \mathrm{GeV}^{6}
$$

to be compared with the QCD value $c_{6}=-4 \pi \alpha_{s}\langle\bar{q} q\rangle^{2} \simeq-1.3 \times 10^{-3} \mathrm{GeV}^{6}$.

In large- $N_{c}$ theories the correlators $\Pi_{V, A}$ can be rewritten as a sum over narrow resonances:

$$
\Pi_{V}=p^{2} \sum_{n} \frac{F_{V_{n}}^{2}}{p^{2}+M_{V_{n}}^{2}}, \quad \Pi_{A}=p^{2} \sum_{n} \frac{F_{A_{n}}^{2}}{p^{2}+M_{A_{n}}^{2}}+F_{\pi}^{2} .
$$

$F_{V_{n}}$ and $F_{A_{n}}$ are the vector and axial-vector decay constants. The poles of $\Pi_{V, A}$ give the mass spectrum. The correlators $\Pi_{V, A}$ calculated via the AdS/CFT correspondence can also be rewritten as in Eq. (2.5). For the vector $n=1$ resonance, the rho meson, we have $M_{\rho} \simeq 2.4 / L_{1}$ that we will use to determine the value of $L_{1} \simeq 1 / 320 \mathrm{MeV}^{-1}$. Then we obtain $F_{V_{1}} \simeq 140 \mathrm{MeV}$. For $\xi \simeq 4$ we obtain $M_{A_{1}} \simeq 1230 \mathrm{MeV}$, that coincides with the mass of $a_{1}$, and $F_{A_{1}} \simeq 160 \mathrm{MeV}$.

\subsection{Vector meson interactions}

The interactions between the different resonances are easily obtained integrating the 5D interactions over $z$ with the corresponding wave-functions. The 5D gauge invariance of the model leads to interesting sum rules among the couplings and masses of the resonances from which we obtain $M_{\rho}^{2} \simeq 3 g_{\rho \pi \pi}^{2} F_{\pi}^{2}, F_{\rho} \simeq \sqrt{3} F_{\pi}$, and the vanishing of the BR of $a_{1}$ into $\pi \gamma$ at the tree-level. Another prediction of the model is the realization of vector meson dominance in the electromagnetic form factor of the pion.

\section{The chiral lagrangian for the PGB}

By integrating all the heavy resonances we can obtain the effective lagrangian for the PGB. This lagrangian is fixed by the chiral symmetry up to some unknown coefficients. Here we give the prediction of our model for these coefficients.

\begin{tabular}{cccccccc}
\hline & $L_{1}$ & $L_{2}$ & $L_{3}$ & $L_{4}$ & $L_{5}$ & $L_{9}$ & $L_{10}$ \\
\hline Exp. & $0.4 \pm 0.3$ & $1.4 \pm 0.3$ & $-3.5 \pm 1.1$ & $-0.3 \pm 0.5$ & $1.4 \pm 0.5$ & $6.9 \pm 0.7$ & $-5.5 \pm 0.7$ \\
\hline AdS $_{5}$ & 0.4 & 0.9 & -2.6 & 0.0 & 1.7 & 5.4 & -5.5 \\
\hline
\end{tabular}

Table 1: Experimental values of the $L_{i}$ at the scale $M_{\rho}$ in units of $10^{-3}$ [7] and the predictions of the AdS $S_{5}$ model for the value $\xi=4$. The coefficients $L_{4,6}$ are zero at the tree-level (leading order in the large- $N_{c}$ expansion), while $L_{7,8}$ will be left for the future ( $L_{7}$ involves the $U(1)$ anomaly and $L_{8}$ receives contributions from the scalar sector).

We also can calculate the electromagnetic pion mass difference in the chiral limit for $\xi=4(5)$ we find $\Delta m_{\pi} \simeq 3.6(4) \mathrm{MeV}$ to be compared with the experimental value $\Delta m_{\pi} \simeq 4.6 \mathrm{MeV}$. 


\section{Conclusions}

We have presented a 5D model that describes some of the properties of QCD related to chiral symmetry breaking. Alike large- $N_{c}$ QCD, this model is defined by a set of infinite weakly coupled resonances. The model depends only on one parameter, $\xi$, related to the quark condensate (apart from the other 3 parameters of the model that are fixed by the 3 parameters that define QCD: the mass gap $\Lambda_{\mathrm{QCD}}, M_{q}$, and $N_{c}$ ). A summary of some of the results is given in Table 1 and Fig. 1 that shows that, within a $30 \%$, they agree with the data.

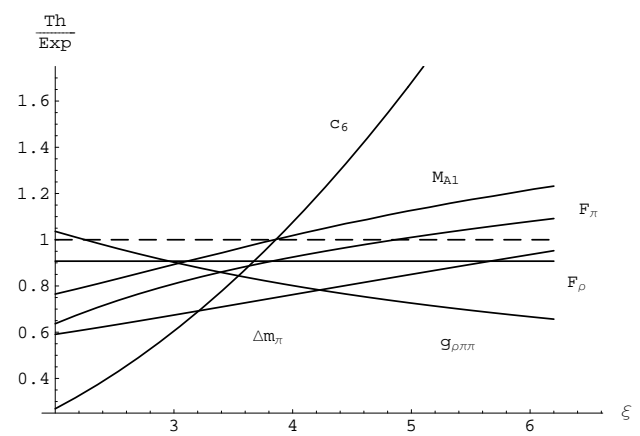

Figure 1: Predictions of the model for some physical quantities as a function of $\xi$ divided by their experimental value. We have taken $M_{q}=0$.

Since the results presented here depend on the $\mathrm{AdS}_{5}$ metric, one can wonder whether the results are robust under possible deviations from AdS. If we want the theory to be almost conformal in the UV, the warp factor for $z \ll L_{1}$ (where $1 / L_{1}$ gives the mass gap) must behave as

$$
a(z) \simeq \frac{L}{z}\left[1+\sum_{i} c_{i}\left(\frac{z}{L_{1}}\right)^{d_{i}}\right],
$$

where $c_{i}$ are numerical constants related to the singlet condensates $\left\langle\mathscr{O}_{i}\right\rangle$ and $d_{i}=\operatorname{Dim}\left[\mathscr{O}_{i}\right]$. In QCD $d_{i} \geq 4$. Eq. (4.1) implies that only for values of $z$ quite close to $L_{1}$ the metric will deviate from AdS. Therefore, unless the coefficients $c_{i}$ are very large, we do not expect large deviations from our results. The $c_{i}$, however, are restricted by the curvature of the space $\mathscr{R}$. We have checked that for $\mathscr{R} \sim 1 / L_{1}^{2}$, our results are not substantially modified by deformations of the metric in the IR.

\section{References}

[1] J. M. Maldacena, Adv. Theor. Math. Phys. 2 (1998) 231.

[2] A. Karch and E. Katz, JHEP 0206 (2002) 043.

[3] L. Da Rold and A. Pomarol, Nucl. Phys. B 721 (2005) 79, arXiv:hep-ph/0510268.

[4] J. Erlich, E. Katz, D. T. Son and M. A. Stephanov, arXiv:hep-ph/0501128.

[5] M. A. Shifman, A. I. Vainshtein and V. I. Zakharov, Nucl. Phys. B 147 (1979) 385; Nucl. Phys. B 147 (1979) 448.

[6] S. S. Gubser, I. R. Klebanov and A. M. Polyakov, Phys. Lett. B 428 (1998) 105; E. Witten, Adv. Theor. Math. Phys. 2 (1998) 253.

[7] A. Pich, arXiv:hep-ph/9806303. 